\title{
PSEUDOBULGES IN BARRED S0 GALAXIES
}

\author{
Peter Erwin ${ }^{1}$, John E. Beckman ${ }^{1}$, and Juan Carlos Vega Beltrán ${ }^{1}$ \\ ${ }^{1}$ Instituto de Astrofísica de Canarias, La Laguna, Tenerife, Spain
}

\begin{abstract}
We present preliminary results from an ongoing study of the bulges of $\mathrm{S} 0$ galaxies. We show that in a subsample of 14 barred S0 galaxies, fully half the photometrically defined bulges show kinematic signatures of pseudobulges - that is, their kinematics are dominated by rotation. In four of these galaxies, we identify at least two subcomponents in the photometric bulge region: flatter, disk or bar components, assocated with disklike kinematics, and rounder "inner bulges," which appear to be hotter systems more like classical bulges.
\end{abstract}

Keywords:

\section{Introduction}

The centers of disk galaxies are conventionally thought to be dominated by a "classical" bulge: a spheroidal, kinematically hot structure similar to a small elliptcal galaxy, which produces the central excess of light above that of the exponential disk. However, Kormendy (1982, 1993) argued that in at least some galaxies the central light came from flattened, disklike components he termed "pseudobulges," possibly the result of bar-driven gas inflow and star formation. At present, very little is known about the demographics of such structures. To address this, we are undertaking a systematic study of bulge morphology and kinematics in early-type disk galaxies.

\section{A High Fraction of S0 Pseudobulges}

We have performed a preliminary analysis for about two-thirds of the barred S0 galaxies in our sample, using long-slit spectroscopy from the ISIS spectrograph of the $4.2 \mathrm{~m}$ WHT and kinematic data from the literature (Kormendy 1982; Simien \& Prugniel 2000; Caon et al. 2000). The results are shown in Figure 1, which plots $V_{\max } / \sigma$ for the photometric bulge regions of fourteen barred S0 galaxies. Half of the galaxies lie on or slightly below the isotropic oblate rotator line, indicating kinematics 
like those of classical bulges or less luminous ellipticals (e.g., Davies et al. 1983). But the other half lie above, making them kinematic pseudobulges. The seven pseudobulges see here imply a lower limit of $30 \%$ for the entire sample of barred S0s. For four of these seven - NGC 2787, NGC 2950, NGC 3945, and NGC 4371 - we have morphological and/or kinematic evidence that the pseudobulge consists of two components (e.g., Erwin et al. 2003): a disklike region (distinct from the galaxy's main, outer disk) and a central region more like a classical bulge.

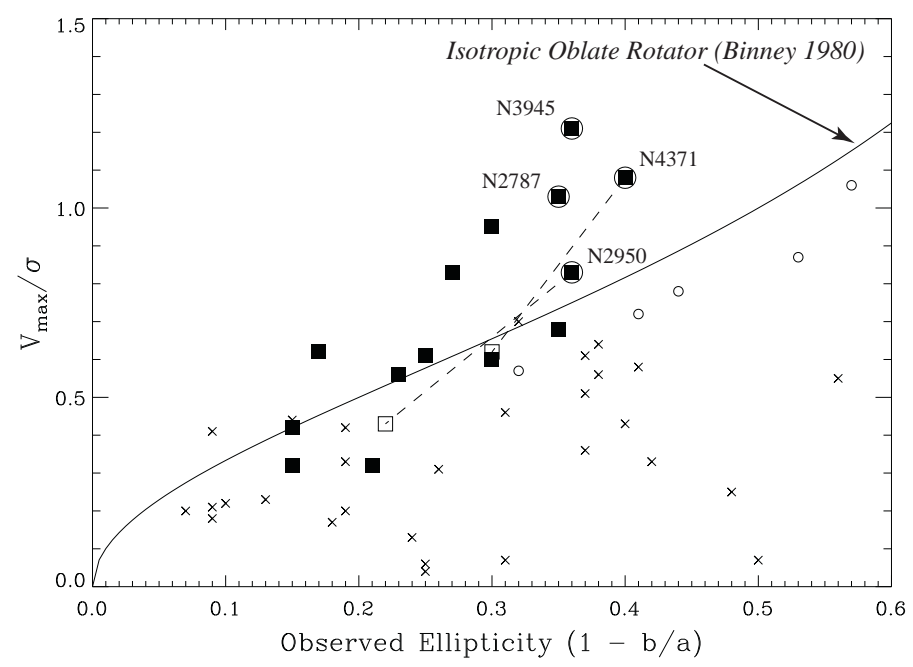

Figure 1. Stellar kinematics of barred S0 bulges: the ratio of maximum velocity $V_{\max }$ to mean velocity dispersion $\sigma$ in the photometric bulge region versus the ellipticity of that region. The solid line is the relation for isotropic oblate rotators (i.e., "classical" bulges and lower-luminosity ellipticals; Binney 1980); galaxies below it are probably dominated by velocity anisotropy, while galaxies above it are dominated by rotation. The crosses are elliptical galaxies from Davies et al. (1983); the open circles are edge-on bulges in early-type disk galaxies (Kormendy \& Illingworth 1982; Jarvis \& Freeman (1985). The barred S0 bulges from our sample are filled boxes. Circled bulges have morphological evidence for multiple components in the photometric bulge region; open boxes indicate kinematics for the innermost regions of NGC 2950 and NGC 4371.

\section{References}

Binney, J. 1980, MNRAS, 190, 421.

Caon, N., Macchetto, D., \& Pastoriza, M. 2000, ApJS, 127, 39.

Erwin, P., Vega Beltrán, J. C., Graham, A. W., \& Beckman, J. E. 2003, ApJ, 597, 929.

Davies, R. L. et al. 1983, ApJ, 266, 41. 
Jarvis, B. J., \& Freeman, K. C., 1985, 295, 324.

Kormendy, J. 1982, ApJ, 257, 75.

Kormendy, J., 1993, in Galactic Bulges, IAU Symposium 153, ed. H. Dejonghe \& H. J. Habing (Dordrecht: Kluwer), 209

Kormendy, J., \& Illingworth, G. 1982, ApJ, 256, 460.

Simien, F., \& Prugniel, Ph. 2000, A\&AS, 145, 263. 